Proyecto de diseño y construcción de una cámara de transporte, tipo tornillo para biomasa forestal

\title{
Proyecto de diseño y construcción de una cámara de transporte, tipo tornillo para biomasa forestal
}

\section{Project design and construction of a transport chamber, screw type for forest biomass}

\section{Projeto de projeto e construção de uma câmara de transporte, tipo parafuso para biomassa florestal}

\author{
Otto F. Balseca-Sampedro I \\ otto.balseca@espoch.edu.ec \\ Jorge I. Caicedo-Reyes II \\ isaias.caicedo@espoch.edu.ec \\ Diego F. Mayorga-Pérez ${ }^{\text {III }}$ \\ d.mayorga@espoch.edu.ec \\ Javier E. Orna-Chávez ${ }^{\text {V }}$ \\ javier.orna@espoch.edu.ec \\ Edwin F. Viteri-Núñez ${ }^{\mathrm{V}}$ \\ eviteri@espoch.edu.ec
}

Recibido: 14 de agosto de 2017 * Corregido: 05 de octubre de 2017 * Aceptado: 17 de noviembre de 2017

I. Magister en Sistemas de Transporte de Petróleo y Derivados, Ingeniero Mecánico, Docente de la Escuela Politécnica de Chimborazo, Riobamba, Chimborazo, Ecuador.

I. Magister en Diseño Mecánico, Ingeniero Mecánico, Docente de la Escuela Politécnica de Chimborazo, Riobamba, Chimborazo, Ecuador.

III. Magister en Seguridad y Prevención de Riesgos del Trabajo, Ingeniero Mecánico, Docente de la Escuela Politécnica de Chimborazo, Riobamba, Chimborazo, Ecuador.

iv. Magister en Diseño Producción y Automatización Industrial, Ingeniero Mecánico, Docente de la Escuela Politécnica de Chimborazo, Riobamba, Chimborazo, Ecuador.

v. Magister en Gerencia de Proyectos de Ecoturismo, Ingeniero Mecánico, Docente de la Escuela Politécnica de Chimborazo, Riobamba, Chimborazo, Ecuador. 


\section{Resumen}

El presente trabajo muestra el proceso de diseño y construcción de un transportador de biomasa forestal para solucionar problemáticas, entre las que resaltan la dificultad de la dosificación de materia prima (madera) y la inseguridad que genera para el operador, quien debe suministrar permanentemente la biomasa durante el proceso para obtener un funcionamiento normal del mismo. Para solucionarlo se desarrolla una cámara de transportación partiendo de la revisión del estado del arte y los desarrollos tecnológicos en el área de transporte y alimentación de desechos sólidos para seleccionar el sistema de alimentación de mejores características, que satisfaga las condiciones de funcionamiento al realizar la selección y diseño de sistemas además de partes basados en la normativa existente, tomando en cuenta además la selección de materiales adecuados. En las pruebas se verifica una mayor estabilidad en el proceso, además de una evidente reducción de la intervención humana.

Palabras clave: biomasa; transportador de tornillo; biomasa forestal; cámara de transportación; gasificador.

\section{Abstract}

This work shows the process of design and construction of a forest biomass transporter to solve problems, among which highlight the difficulty of the dosage of raw material (wood) and the insecurity that it generates for the operator, who must permanently supply the biomass during the process to obtain normal operation of it. To solve this, a transportation chamber is developed based on a review of the state of the art and technological developments in the area of solid waste transport and feeding to select the best characteristics feeding system that satisfies the operating conditions when making the selection. and design of systems as well as parts based on existing regulations, taking into account also the selection of suitable materials. In the tests, a greater stability in the process is verified, in addition to an evident reduction in human intervention.

Keywords: biomass; screw conveyor; forest biomass; transportation camera; gasifier.

\section{Resumo}

Este trabalho mostra o processo de projeto e construção de um transportador de biomassa florestal para resolver problemas, dentre os quais destacam a dificuldade da dosagem de matéria-prima (madeira) e a insegurança que gera para o operador, que deve fornecer permanentemente a biomassa durante o processo para obter o seu funcionamento normal. Para resolver isso, uma câmara de 
Proyecto de diseño y construcción de una cámara de transporte, tipo tornillo para biomasa forestal

transporte é desenvolvida com base em uma revisão do estado da arte e dos desenvolvimentos tecnológicos na área de transporte de resíduos sólidos e alimentação para selecionar o melhor sistema de alimentação de características que satisfaça as condições de operação ao fazer a seleção. e design de sistemas, bem como peças baseadas em regulamentos existentes, levando em consideração também a seleção de materiais adequados. Nos testes, verifica-se uma maior estabilidade no processo, além de uma redução evidente na intervenção humana.

Palavras chave: biomassa; transportador de parafuso; biomassa florestal; câmera de transporte; gasificador.

\section{Introducción}

Existe diversidad de mecanismos para la transportación de productos, los cuales se manejan bajo las diferentes necesidades de un proceso, es decir que la elección del mecanismo dependerá de los diferentes parámetros que intervienen en un determinado proceso. (Morales, 2010)

Un sistema de transportación es un conjunto de procesos y operaciones, que tienen como función entregar la cantidad correcta de producto, con la participación mínima y a veces casi nula del personal humano. La producción se obtiene en porciones, cuando se completa la porción el equipo debe volver a llenar producto y procesar la siguiente porción. (Morales, 2010)

Los transportadores de sólidos volumétricos son utilizados en diferentes ámbitos, siendo los transportadores de tornillo los equipos más sencillos y económicos que existen para trasegar sólidos de un lado a otro de cualquier instalación. El mecanismo empleado es un tornillo sinfín cuyo diámetro se dimensiona en función de los requerimientos de transporte de producto. Su empleo resulta óptimo en aplicaciones en las que se requieren movimientos de grandes volúmenes a distancias relativamente cortas. (TECNIDOS: Sistemas y Procesos, 2014)

A nivel mundial los dosificadores son utilizados generalmente en el ámbito alimenticio, en Colombia, por ejemplo, la Federación Nacional de Cafeteros de Colombia, ha instalado un separador hidráulico de tolva y tornillo sinfín en Cenicafé con el fin de mejorar la calidad de la materia prima que llega a los comederos y evitar daños a las máquinas que hacen parte del proceso. En este dispositivo hidromecánico se remueve por flotación material liviano que está conformado por frutos de café defectuosos, como frutos vanos, muy brocados, secos o provenientes de plantas enfermas, entre otros, 
lo mismo que impurezas livianas como hojas y trozos de ramas. Se obtienen varias ventajas tras la instalación del transportador como, por ejemplo: control del flujo de café a la despulpadora, que se traduce en eficiencia del proceso; disminución de café con daño mecánico por mejor funcionamiento de los equipos; disminución de los daños a los equipos por remover los objetos duros y densos. (Sanz, y otros, 2014)

Otro claro ejemplo del uso de este tipo de transportador se presenta en un estudio realizado por el Instituto Politécnico Nacional de la ciudad de México DF, en el que se desarrolla un sistema de transportación automático de alimentos para equinos basado en el principio de tornillo sin fin, obteniendo resultados favorables como la precisión que se tiene en cuanto a la dosis de alimento que se suministra a cada caballo además de un ahorro de energía eléctrica y la minimización del espacio que implica la actividad alimenticia de caballos en un hípico pudiendo verse así las virtudes de un comedero automático. (Franco, y otros, 2010)

De igual manera en la ciudad de Pereira, se presenta el diseño y construcción de un prototipo de un dosificador de alimento concentrado para cerdos, como respuesta a las falencias que se presentan en la alimentación de porcinos, obteniendo como resultado una mejora en el manejo de dicho proceso ya que se aprovecha al máximo cada bulto de concentrado, evitando desperdicios, aumentando la posibilidad de una alimentación adecuada, disminuyendo horas de trabajo en el operario e incrementando la eficiencia a la par que se tecnifica el método de alimentación. (Osipna, y otros, 2013)

En el proyecto: "Diseño y montaje de un sistema dosificador para galletas tipo cracker fermentadas" de la Universidad Tecnológica de Pereira se menciona varios aspectos muy importantes como resultado de la investigación, entre los que se destaca la comprobación de que implementar sistemas automatizados de transportación, reduce el tiempo de manipulación del producto por lo que la probabilidad de contaminación es menor, además de afirmar que al reducir la carga laboral de los operarios también se reduce el estrés, lo que permite que el trabajador este más relajado teniendo inclusive tiempo suficiente para realizar actividades de mantenimiento como es el aseo de la máquina y del puesto de trabajo. (Orozco, y otros, 2016)

En el Ecuador al igual que en el resto del mundo el uso de dosificadores se lo ha implementado también en el área agrícola, como lo muestra el proyecto: "Diseño y construcción de un dosificador 
de tres productos en forma secuencial", mismo que se enfoca en la distribución de granos, en dicha investigación se menciona que los tornillos evacuadores son los elementos más importantes en los dosificadores de sólidos ya que éstos proporcionan el grano evacuado, y éste determina la precisión máxima que se puede obtener en el proceso. (Rivera, 2002)

También es importante hacer referencia a la investigación desarrollada en la Escuela Superior Politécnica Nacional sobre el diseño y simulación de una planta dosificadora automática de suplemento nutricional para ganado lechero, en el que se diseña un dosificador según las necesidades y los establecimientos destinados para el ordeño, siendo fundamental que el ruido emitido sea mínimo, en las pruebas realizadas se menciona la importancia de regular la velocidad de transportación, ya que la misma determinará el adecuado funcionamiento del dosificador. (Carrillo, 2015)

Este trabajo tiene como objetivo presentar el desarrollo de la cámara de transportación para biomasa forestal.

\section{Metodología}

El desarrollo de la cámara de transportación para biomasa forestal se basa en los parámetros establecidos por la norma CEMA, "Estándares de Diseño de Transportadores” y las especificaciones y requerimientos que determina el usuario de este proyecto.

Analizando los resultados obtenidos en el diagrama QFD o casa de calidad, se puede decir que los requerimientos técnicos más importantes son los siguientes:

- Flujo másico. El transportador debe ser capaz de alimentar continuamente una cantidad de $25.71 \mathrm{~kg} / \mathrm{h}$, para abastecer la necesidad de biomasa forestal.

- La economía. El transportador debe prestar características técnicas de seguridad, pero teniendo en cuenta el costo final de la máquina, ya que este factor hace que el producto sea didáctico frente a otras máquinas.

- Programación. Una buena programación permite que se realice una eficiente transportación, cuidando de utilizar los mejores elementos para obtener dichos resultados.

- $\quad$ Material. Se construye según las funciones que desempeñe cada parte del mecanismo. 
Proyecto de diseño y construcción de una cámara de transporte, tipo tornillo para biomasa forestal

Los criterios para la selección del motoreductor son la potencia necesaria para llevar a cabo la transportación de biomasa y la velocidad de giro, la velocidad de giro necesaria es muy baja (menor a $5 \mathrm{rpm}$ ), pero es extremadamente difícil alcanzar dicha velocidad con un motoreductor por lo cual el sistema debe ser acoplado a un variador de frecuencia para alcanzar velocidades bajas.

Para el presente proyecto se selecciona un motoreductor de 1 HP con una velocidad máxima de giro de 30 RPM.

Para el transporte de biomasa se determina la aplicación de un transportador de tornillo sin fin, que es el elemento mecánico que consta de un eje sobre el cual se desarrolla una espiral, la cual transporta materiales ya sea horizontalmente, verticalmente o en una pendiente.

El tornillo sinfín debe ser capaz de transportar cubos de madera de hasta $50 \mathrm{~mm}$ de lado. Estableciendo que las dimensiones máximas de las probetas de biomasa forestal que van a ingresar en el transportador no deberán superar los $10 \mathrm{~cm}$.

El tornillo sinfín debe ser capaz de transportar cubos de madera de hasta $50 \mathrm{~mm}$ de lado, para realizar pruebas de funcionamiento en el gasificador tipo Downdraft del laboratorio de térmicas de la Facultad de Mecánica.

Para determinar las dimensiones del transportador se requieren las dimensiones máximas de los cubos a ser transportados, mediante la ecuación:

$$
L_{\max }=\sqrt{3} L
$$

Esta ecuación se determina mediante la aplicación del teorema de pitágoras para hallar la dimensión máxima de un cubo de lado 1.

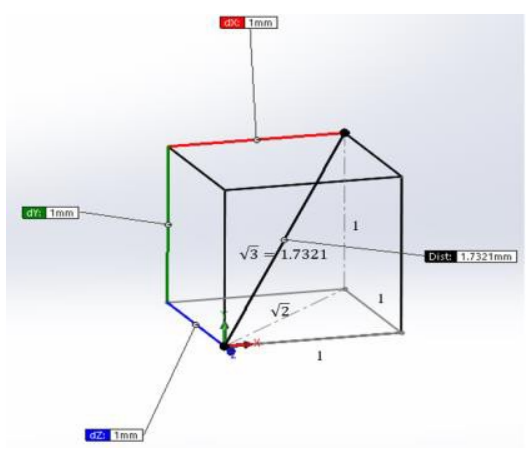


Figura 1. Dimensiones máximas de un cubo

De la figura 1 se puede decir que la dimensión máxima de un cubo es raíz de 3 veces su lado, como se indica en la ecuación:

$$
L_{\max }=\sqrt{3} *(5 \mathrm{~cm})=8.66 \mathrm{~cm}
$$

Para el diseño del tornillo sinfín, para evitar un posible atascamiento se va a trabajar con un factor de seguridad de 1.15 en el sobredimensionamiento de los cubos de biomasa.

$$
L_{\max }=1.15 * 8.7=10.005 \mathrm{~cm}
$$

Por lo tanto las dimensiones máximas de las probetas que van a ingresar en el dosificador de biomasa no deberán superar los $10 \mathrm{~cm}$.

Para las pruebas de funcionamiento no se deberán usar probetas de más de $10 \mathrm{~cm}$ de longitud máxima, la opción para el dimensionamiento del tornillo sinfín es determinar en función del tamaño máximo de las partículas a ser transportadas según el estándar de diseño de transportadores CEMA.

Como se muestra en la figura 2 del catálogo de selección de transportadores helicoidales se procede a determinar el diámetro externo del tornillo sin fin y el diámetro del eje para obtener el espacio libre entre el eje y el extremo de las hélices.

\begin{tabular}{|c|c|c|c|c|c|c|c|c|c|c|}
\hline $\begin{array}{l}\text { Conveyor } \\
\text { Diameter } \\
\text { Inches }\end{array}$ & $\begin{array}{l}\text { Shaft and } \\
\text { coupling } \\
\text { Diameter }\end{array}$ & A & B & c & D & E & $\mathrm{F}$ & G & $H$ & J \\
\hline 6 & $11 / 2$ & $9^{\prime}-10^{\prime \prime}$ & $10^{\prime}-0^{\circ}$ & 2 & 6 & 7 & 5 & $41 / 2$ & $55 / 8$ & $11 / 2$ \\
\hline \multirow[t]{2}{*}{9} & $11 / 2$ & $9^{\prime}-10^{*}$ & $10^{\circ}-0^{*}$ & 2 & 8 & 10 & $71 / 8$ & $61 / 8$ & $77 / 8$ & $15 / 8$ \\
\hline & 2 & $9^{\prime}-10^{\circ}$ & $10^{\prime}-0^{\circ}$ & 2 & 8 & 10 & $71 / 8$ & $61 / 8$ & $77 / 8$ & $15 / 8$ \\
\hline 10 & $11 / 2$ & $9^{\prime}-10^{*}$ & $10^{\circ}-0^{-0^{*}}$ & 2 & 9 & 11 & $71 / 8$ & $63 / 8$ & $87 / 8$ & $13 / 4$ \\
\hline \multirow{3}{*}{12} & 2 & $\begin{array}{c}9^{\prime}-10^{10} \\
11^{*}\end{array}$ & $\begin{array}{l}10^{2}-0^{-1} \\
12-0^{0}\end{array}$ & 2 & ${ }_{109}^{9}$ & $\begin{array}{l}11 \\
13\end{array}$ & $\begin{array}{l}71 / 8 \\
8788\end{array}$ & $\begin{array}{l}63 / 8 \\
73 / 4\end{array}$ & $\begin{array}{l}8778 \\
95 / 8\end{array}$ & $13 / 4$ \\
\hline & $27 / 16$ & $11^{-1}-g^{-9}$ & $12^{-2}-0^{-0}$ & 3 & $\begin{array}{l}101 / 2 \\
101 / 2\end{array}$ & $\begin{array}{l}73 \\
13\end{array}$ & $\begin{array}{l}878 \\
8718\end{array}$ & $\begin{array}{l}73 / 4 \\
73 / 4\end{array}$ & $\begin{array}{l}95 / 8 \\
95 / 8\end{array}$ & $\begin{array}{l}2 \\
2\end{array}$ \\
\hline & & $11-g^{-9}$ & $12^{-}-0^{-0}$ & 3 & $\begin{array}{l}101 / 2 \\
10\end{array}$ & 13 & $\begin{array}{l}8718 \\
8718\end{array}$ & $73 / 4$ & $\begin{array}{l}95 / 8 \\
95 / 8\end{array}$ & 2 \\
\hline \multirow[t]{2}{*}{14} & $27 / 16$ & $11-9$ & $12^{-1} 0^{\circ}$ & 3 & $\begin{array}{ll}011 / 2 \\
11\end{array}$ & 15 & $\begin{array}{l}101 / 8 \\
\end{array}$ & $\begin{array}{l}91 / 4 \\
91 / 4\end{array}$ & $107 / 8$ & 2 \\
\hline & 3 & $119^{-9}$ & $12^{-}-0^{-}$ & 3 & $111 / 2$ & 15 & $\begin{array}{l}101 / 8 \\
\end{array}$ & $\begin{array}{l}91 / 4 \\
91 / 4\end{array}$ & $107 / 8$ & 2 \\
\hline 16 & 3 & $11^{\prime}-9^{-1}$ & $12^{\prime}-0^{\circ}$ & 3 & $131 / 2$ & 17 & $111 / 8$ & $105 / 8$ & 12 & $21 / 2$ \\
\hline \multirow[t]{2}{*}{18} & 3 & $11-g^{\prime \prime}$ & $12^{2}-0^{\circ}$ & 3 & $141 / 2$ & 19 & $123 / 8$ & $121 / 8$ & 13 3/8 & $21 / 2$ \\
\hline & $37 / 16$ & $11-8^{8}$ & $12^{2}-0^{\circ}$ & 4 & $\begin{array}{ll}141 / 2 \\
15\end{array}$ & 19 & $123 / 8$ & $121 / 8$ & $133 / 8$ & $21 / 2$ \\
\hline 20 & $\begin{array}{c}3 \\
37 / 11\end{array}$ & 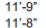 & $\begin{array}{l}12-0^{-0} \\
12-0^{0}\end{array}$ & $\begin{array}{l}3 \\
4\end{array}$ & $\begin{array}{l}151 / 2 \\
1512\end{array}$ & $\begin{array}{l}21 \\
21\end{array}$ & $\begin{array}{l}133 / 8 \\
133 / 8\end{array}$ & $\begin{array}{l}131 / 2 / 2 \\
131 / 2\end{array}$ & $\begin{array}{l}15 \\
15\end{array}$ & $\begin{array}{l}21 / 2 \\
21 / 2\end{array}$ \\
\hline 24 & $37 / 16$ & $111^{-8}$ & $12^{2}-0^{\circ}$ & 4 & $171 / 2$ & 25 & $153 / 8$ & $\begin{array}{l}161 / 2 \\
16\end{array}$ & $181 / 8$ & $\begin{array}{l}21 / 2 \\
21 / 2\end{array}$ \\
\hline
\end{tabular}

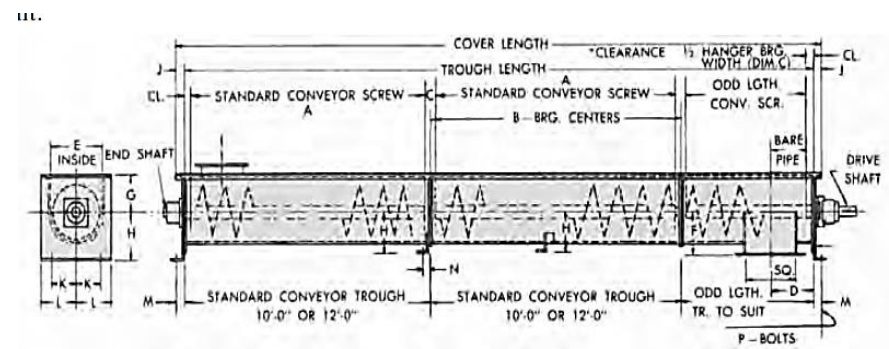

Figura 2. Dimensiones principales del transportador según Catálogo Screw Conveyor 
Proyecto de diseño y construcción de una cámara de transporte, tipo tornillo para biomasa forestal

Las dimensiones seleccionadas corresponden a un diámetro externo del transportador de 10 pulgadas y un diámetro del eje de 2 pulgadas lo cual nos deja como espacio libre 8 pulgadas que son aproximadamente igual a $20.32 \mathrm{~cm}$ a cada lado del transportador.

Es importante comprobar si el espacio libre es mayor o igual a las dimensiones máximas de las probetas a ser dosificadas. Si la condición se cumple entonces se ha realizado un correcto dimensionamiento del dosificador de biomasa.

$$
\frac{20.32}{2}=10.16 \mathrm{~cm}>L_{\max }=10.005 \mathrm{~cm}
$$

Los resultados del dimensionamiento del tornillo sinfín se muestran en la Tabla 1:

\begin{tabular}{|l|}
\hline Resultados dimensionamiento \\
\hline Diámetro externo: $250 \mathrm{~mm}=10 \mathrm{in}$ \\
Diámetro del eje: $50 \mathrm{~mm}=2$ in \\
Paso: $250 \mathrm{~mm}=10 \mathrm{in}$ \\
Longitud del eje: $150 \mathrm{~cm}=6$ in \\
Longitud de hélices: $100 \mathrm{~cm}=4$ in \\
\hline
\end{tabular}

Tabla 1 Dimensionamiento Tornillo

Las dimensiones anteriores permiten la construcción del sinfín con un espesor de $3 \mathrm{~mm}$, debido a la rigidez de la soldabilidad requeridas. La longitud del tornillo se asume en base al espacio disponible.

Para asegurar un flujo másico de 25.71 kilogramos por cada hora, se va a trabajar con la tabla de capacidad del dosificador con un porcentaje del 15 por ciento de material con respecto a la capacidad del tubo exterior del dosificador, como se indica en la figura 3.

\begin{tabular}{|c|c|c|c|c|c|c|}
\hline \multirow{2}{*}{\multicolumn{2}{|c|}{$\begin{array}{l}\text { Material Class } \\
\text { Code }\end{array}$}} & \multirow{2}{*}{$\begin{array}{c}\text { Degree of } \\
\text { Trough Loading }\end{array}$} & \multirow{2}{*}{$\begin{array}{c}\text { Screw } \\
\text { Dia. } \\
\text { Inch. }\end{array}$} & \multirow{2}{*}{$\begin{array}{c}\text { Maximum } \\
\text { RPMI }\end{array}$} & \multicolumn{2}{|c|}{$\begin{array}{l}\text { Capacity, Cubic } \\
\text { Feet Per Hour }\end{array}$} \\
\hline & & & & & $\begin{array}{l}\text { At Max. } \\
\text { RPMI }\end{array}$ & At One RPM \\
\hline $\begin{array}{l}\text { A-17 } \\
\text { A-27 } \\
\text { A-37 } \\
\text { A- } 47 \\
\text { B-17 } \\
\text { B-27 } \\
\text { B-37 } \\
\text { B- } 47 \\
\text { C-17 } \\
\text { C- } 27 \\
\text { - } 37 \\
\text { - } 47\end{array}$ & $\begin{array}{l}\text { D-17 } \\
\text { D-27 } \\
\text { D-37 } \\
D-47 \\
\text { E-17 } \\
\text { E-27 } \\
\text { E-37 } \\
\text { - } 47\end{array}$ & $15 \%$ & $\begin{array}{l}6 \\
9 \\
12 \\
14\end{array}$ & $\begin{array}{l}60 \\
55 \\
50 \\
50\end{array}$ & $\begin{array}{c}700 \\
1,010 \\
1,250 \\
2,180\end{array}$ & $\begin{array}{l}0.75 \\
2.72 \\
6.46 \\
10.4\end{array}$ \\
\hline
\end{tabular}

Figura 3. Capacidad 
Proyecto de diseño y construcción de una cámara de transporte, tipo tornillo para biomasa forestal

Es necesario obtener la capacidad a una RPM con el diámetro del tubo de 10 pulgadas para lo cual se trabaja mediante una interpolación, obteniéndose una capacidad de 3,96 pies cúbicos por cadahora.

Mediante la densidad de la madera se puede obtener un valor tentativo de flujo másico, como se indica en la siguiente ecuación:

$$
\rho=\frac{f_{m}}{f_{v}}
$$

Realizando el despeje anterior se obtiene que a una velocidad angular de una RPM se puede obtener un flujo másico de 98 kilogramos por cada hora, pero al no existir la madera en forma de cubos en la clasificación del material para el diseño de dosificadores en el manual de la norma CEMA. Se debe procede a realizar una medición experimental en la cual se requiere de un mecanismo de control de capacidad del dosificador y además de un variador de velocidad del eje de transmisión.

El tubo exterior del tornillo sinfín fue dimensionado anteriormente donde se determinó que el diámetro exterior del tubo exterior del tornillo transportador es de 10 pulgadas. Para determinar su espesor se requiere determinar las cargas a las cuales se encuentra sometido.

La carga que va a soportar el tubo del dosificador de biomasa es de los $154.26 \mathrm{~kg}$, calculados con anterioridad.

Para hallar el peso de la biomasa almacenada en la biomasa se trabaja con la siguiente ecuación:

$$
w=m_{\text {biomasa }} g=154.26(9.8)=1512 \mathrm{~N}
$$

La distancia entre apoyos es de $1.5 \mathrm{~m}$, mientras que la distancia desde el apoyo al punto de aplicación de la carga es de $0.2 \mathrm{~m}$, para ello se asume que la carga de la biomasa almacenada se aplica desde el centro de la tolva.

A continuación, se indican los diagramas de cuerpo libre, fuerza cortante y momento flector para el tubo externo del dosificador de biomasa. (Figura 4) 
Proyecto de diseño y construcción de una cámara de transporte, tipo tornillo para biomasa forestal

Figura 4. a) Diagrama de cargas del tubo exterior del dosificador de biomasa.
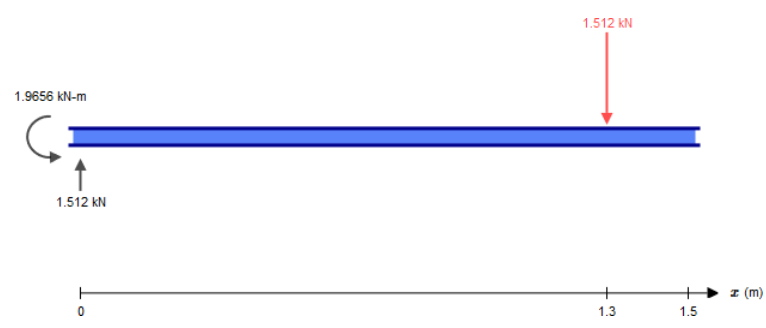

b) Diagrama de fuerza cortante

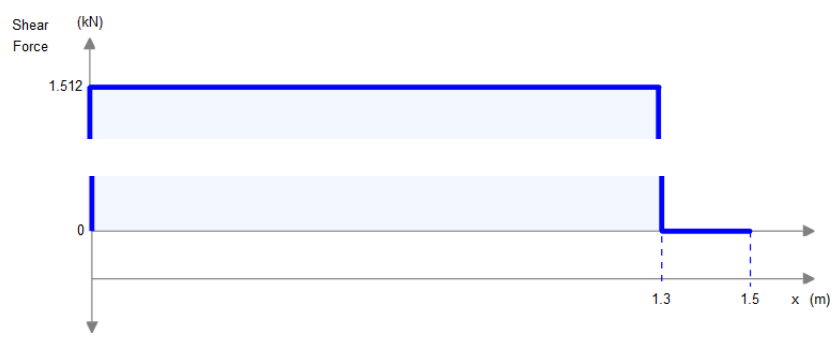

c) Diagrama de momento flector

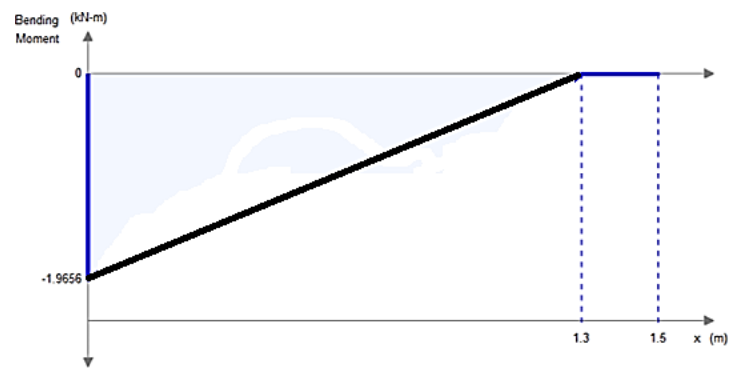

Para determinar si el tubo exterior del dosificador resiste la carga de la madera ubicada en la tolva debemos asumir el material y el espesor en función de la facilidad de obtener la plancha en el mercado para su posterior embarolado.

Un material con excelentes propiedades mecánicas para ser embarolado es una plancha de acero A36 ya que cuenta con un $25 \%$ de porcentaje de alargamiento y con una resistencia mecánica muy aceptable. (Figura 5) 


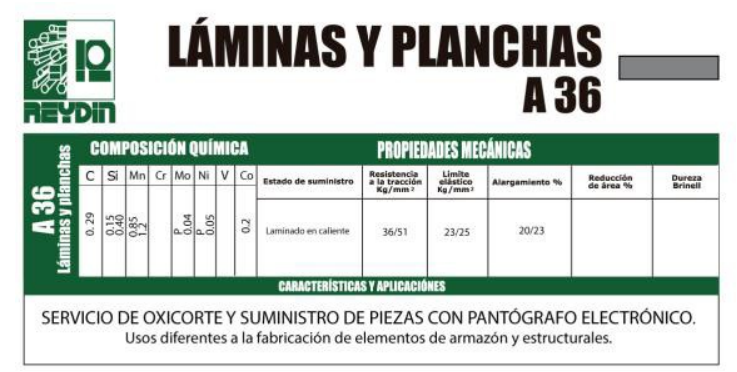

Figura 5. Propiedades mecánicas de acero A36

El espesor que se asume para los posteriores cálculos es de $3 \mathrm{~mm}$, dicho espesor va a usarse para determinar el esfuerzo al cual está sometido el tubo del transportador. Y dicho espesor debe ser menor al esfuerzo admisible para el Acero A36.

Para realizar la verificación del tubo exterior del dosificador de biomasa se requiere utilizar la siguiente ecuación:

$$
\sigma=\frac{M_{f} y}{I_{x}}
$$

En necesario realizar el cálculo del momento de inercia con la fórmula que se indica a continuación:

$$
I_{x}=\frac{\pi}{4}\left(R^{4}-r^{4}\right)
$$

El esfuerzo anterior debe ser comparado con el esfuerzo admisible del acero A36.

\begin{tabular}{|l|c|}
\hline \multicolumn{1}{|c|}{ Propiedad } & Valor \\
\hline Densidad, g/cm & 7.85 \\
\hline $\begin{array}{l}\text { Resistencia a la tracción, } \\
\text { MPa }\end{array}$ & $400-552$ \\
\hline Límite de Fluencia, MPa & 250 \\
\hline Módulo de Young, MPa & 200000 \\
\hline Modulo de Cortante, MPa & 79300 \\
\hline Coeficiente de Poisson & 0.26 \\
\hline Elongación, \% & 18 \\
\hline Dureza Rockwell B & $67-83$ \\
\hline Dureza Brinell & $119-159$ \\
\hline
\end{tabular}

Figura 6. Propiedades mecánicas ASTM A36

Como se indica en la figura 6 el límite de fluencia del acero A36 es:

$$
\sigma_{a d m}=250 \mathrm{MPa}
$$


Por lo tanto con el esfuerzo conocido y el esfuerzo admisible es posible determinar el factor de seguridad:

$$
n=\frac{\sigma_{a d m}}{\sigma}=\frac{250 M P a}{13.184}=18.96
$$

El factor de seguridad es muy alto con respecto al valor permisible $(n>1)$, pero es necesario determinar primero la resistencia de la soldadura de brida de acoplamiento del transportador de biomasa con el gasificador. El cálculo de la brida se realiza en el siguiente apartado.

El eje central es un elemento cilíndrico de sección circular, que puede esta fijo o girando, sobre el que se montan engranajes, poleas, volantes, ruedas de cadena, manivelas o manubrios, así como otros elementos mecánicos de transmisión de fuerza o potencia, los ejes de transmisión son barras sometidas a cargas de flexión, tensión, compresión o torsión que actúan individualmente o combinadas.

Para el caso del transportador de biomasa el eje se debe mantener en movimiento, el eje va a estar sometido principalmente al torque que genera la resistencia de la biomasa de la tolva.

El eje se diseña estáticamente y dinámicamente, se conoce como datos la carga a la cual va a estar sometido el sistema de alimentación, y la velocidad angular.

Para el diseño se considera que se encuentra acoplado a un sistema de transmisión por engranajes a un motor eléctrico que transmite la potencia necesaria al tornillo sin fin apoyado entre dos rodamientos los mismos que absorben la fuerza radial.

La carga del alimento se distribuye de manera uniforme, el peso de la biomasa del tornillo sin fin se obtiene a partir de la siguiente ecuación:

$$
Q=\frac{P p}{L}
$$

Dicha carga distribuida está aplicada a lo largo del eje y tiene un valor de $19.77 \frac{\mathrm{kg}}{\mathrm{m}}$

Mediante mediciones experimentales se pudo obtener que para vencer la inercia tanto del eje así como de la biomasa a ser transportada se requiere de un momento torsor de $200 \mathrm{Nm}$.

Las cargas se lo representan en la figura 7, y con la ayuda del software MDSOLIDS, se puede determinar estas reacciones: 
Proyecto de diseño y construcción de una cámara de transporte, tipo tornillo para biomasa forestal

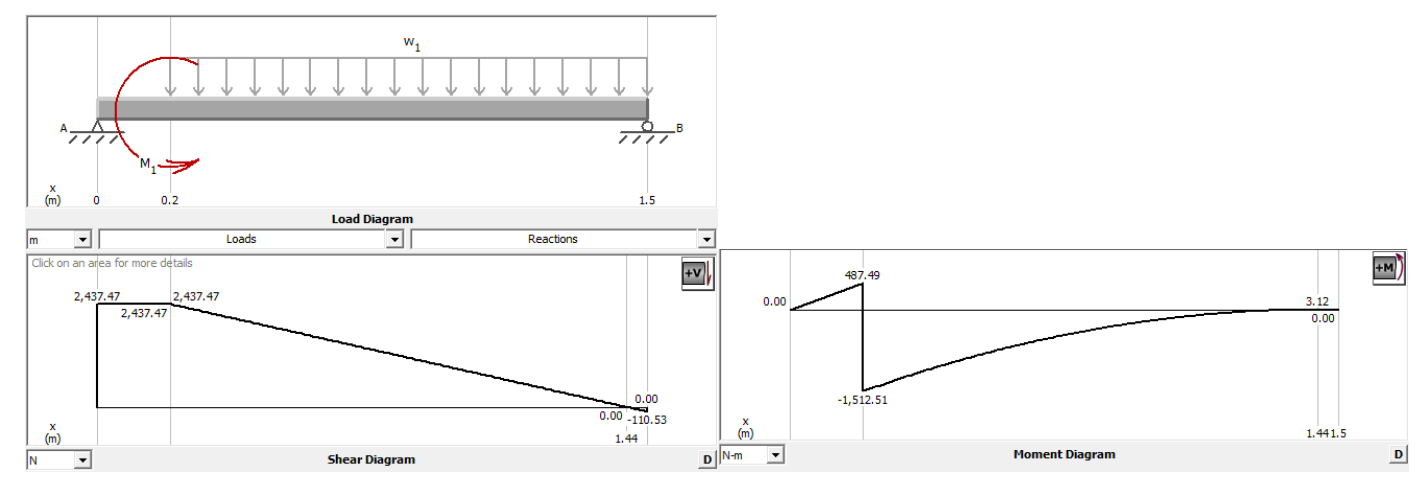

Figura 7. Diagrama de cargas X - Y

El momento flector se encuentra en el plano X - Y según el eje de referencia que se asume y es igual a:

$$
M_{f}=1512,51 \mathrm{Nm}
$$

Material base del eje

En muchos casos se necesita determinar la resistencia de piezas correspondiente a estados de esfuerzo diferentes. En muchos casos los esfuerzos fluctúan sin pasar por cero. En la figura 8 se muestra la relación esfuerzo - tiempo que se puede presentar.

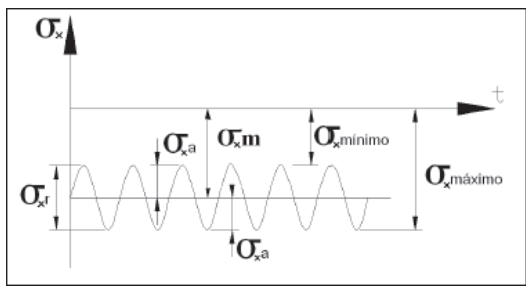

Figura 8. Relación esfuerzo - tiempo. Esfuerzos medios y alternantes.

Se ha seleccionado para el eje de transmisión un acero AISI 1045, el mismo que posee buenas características de resistencia mecánica. Las propiedades mecánicas del acero 1045 son:

$$
\begin{gathered}
S_{u t}=500 \mathrm{MPa} \\
S_{y}=250 \mathrm{MPa}=250000000 \frac{\mathrm{N}}{\mathrm{m}^{2}}
\end{gathered}
$$


Proyecto de diseño y construcción de una cámara de transporte, tipo tornillo para biomasa forestal

$$
\left.d_{\min }=\frac{32 * n}{\pi * S_{y}} \quad\left(\mathbb{M}+T^{2}\right)^{\frac{1}{2}}\right]^{\frac{1}{3}}
$$

Diseño a fatiga

Considerando la ecuación de fatiga de materiales se tiene:

$$
S_{e}=S_{e}^{*} K_{a}^{*} K * \underset{b}{K} * \underset{c}{K} * K_{d} \quad e
$$

Para aceros:

$$
\begin{gathered}
S_{e}^{\prime}=0,5 S_{u t}=250 \mathrm{MPa} \\
K_{f}=1+q *\left(k_{t}-1\right)
\end{gathered}
$$

Al resolver la ecuación se tiene:

$K_{f}=1.3$

$K_{e}=0.8$

Resuelta la ecuación se tiene:

$$
S_{e}=180 M P a
$$

El eje está sometido a flexión y torsión por lo tanto:

$$
\begin{array}{r}
2 \propto=\tan ^{-1}\left(\frac{M I \Delta_{u t}}{M_{t} S_{e}}\right) \\
\propto=43.63
\end{array}
$$

$$
\begin{gathered}
\sigma_{a}=\frac{32 M \operatorname{sen}(2 \alpha)}{\pi d^{3}}=123 \mathrm{MPa} \\
\sigma_{m}=\frac{32 T \cos (2 \alpha)}{\pi d^{3}}=776 \mathrm{MPa}
\end{gathered}
$$




$$
\begin{aligned}
& \frac{\sigma_{a}}{S_{e}}+\frac{\sigma_{m}}{S_{u t}}=\frac{1}{n} \\
& n=1.2
\end{aligned}
$$

El valor obtenido del factor de seguridad garantiza que el eje de transmisión no falle por fatiga.

Para el dimensionamiento del espesor de las aspas de la hélice se considera a las hélices como un anillo soportando la carga de arrastre de la biomasa forestal. Además, que independientemente del cálculo del espesor subsiguiente es importante conocer que, para la construcción de dicha hélice, existe un espesor mínimo de $3 \mathrm{~mm}$ para obtener la rigidez suficiente luego de la deformación de la plancha mediante torneado. Como se indica es importante tener una excelente rigidez para evitar la fuga del gas pobre entre el tubo exterior del transportador y los bordes de la hélice.

Además de que se observa que existen dos fuerzas principales que actúan en una junta tipo brida. A continuación, se indican las cargas conocidas:

- Presión de funcionamiento del gasificador: 1 Atmósfera $=14.7$ psi

- La carga de separación se la puede determinar a partir de la siguiente relación:

$$
P=\frac{Q}{n_{1} \eta}
$$

Si la velocidad del eje es de 3 RPM cada hora gira 180 revoluciones, por lo tanto:

$$
P=\frac{25.71 \mathrm{~kg}}{n_{1} \mathrm{n}_{2}}=0.028 \mathrm{~kg}=0.28 \mathrm{~N}
$$

El momento al cual está sometida cada aspa se la puede determinar con la altura de cada aspa la cual en el dimensionamiento del transportador se lo determina como:

$$
\begin{gathered}
H_{\text {aspa }}=100 \mathrm{~mm}=0.1 \mathrm{~m} \\
M=H_{\text {aspa }} * P=0.028 \mathrm{Nm}
\end{gathered}
$$


Para determinar la carga concentrada en el aspa de la hélice, tomando en cuenta que la junta soldada actúa como el apoyo. Se utiliza la siguiente ecuación:

$$
\begin{aligned}
& p=\frac{M_{f}}{r} \\
& p=\frac{M_{f}}{(n)^{2} r}=\frac{0.028 \mathrm{Nm}}{(25) 0.075 \mathrm{~m}}=0.014 \mathrm{~N}
\end{aligned}
$$

Luego de determinar la carga de la soldadura con la solicitación de carga más elevada se va a realizar el cálculo para determinar el espesor de la brida, como un elemento sometido a flexión pura.

Para determinar los diagramas de fuerza cortante y momento flector es importante asumir que el anillo de la brida se comporta como un elemento sometido a flexión con los siguientes datos:

Carga puntual: $0.014 \mathrm{~N}$, aplicada en el centro del anillo.

Distancia entre apoyos, es el perímetro de la circunferencia del anillo.

$$
\text { perímetro: } \pi(0.075 \mathrm{~m})=0.235 \mathrm{~m}
$$

El momento máximo se lo puede determinar con la siguiente ecuación:

$$
\begin{gathered}
M=p \frac{\text { perimetro }}{2} \\
M=p \frac{\text { perimetro }}{2}=0.014 * \frac{0.235}{2} \mathrm{Nm}=0.0016 \mathrm{Nm} \\
M_{f}=0.0016 \mathrm{Nm} \\
I_{x}=\frac{1}{12} b h^{3}
\end{gathered}
$$

La base es la diferencia entre diámetro interno y externo del anillo, mientras que la altura es el espesor, el cual se puede asumir y ser igual a $3 \mathrm{~mm}$ para facilitar la soldadura de a brida con el tubo exterior.

En el apartado anterior se determinó un elevado factor de seguridad para el diseño del tubo exterior, pero para el diseño de la brida se tiene: 
Proyecto de diseño y construcción de una cámara de transporte, tipo tornillo para biomasa forestal

$$
n=\frac{\sigma_{a d m}}{\sigma}
$$

Como se mencionó anteriormente la función principal de las hélices será tener una estabilidad dimensional para evitar las fugas de gas pobre, y por ello se mantiene el espesor de $3 \mathrm{~mm}$ seleccionado anteriormente.

Se realiza la selección de rodamientos necesarios para el correcto funcionamiento de la máquina. Se requiere el cálculo de la reacción radial mayor sobre los apoyos mediante la siguiente relación:

$$
R_{r}=\sqrt{\left(R_{A}^{2}+R_{B}^{2}\right)_{B}}
$$

Entonces:

$$
\begin{gathered}
R_{A}=2437 N \\
R_{B}=-110 \mathrm{~N}
\end{gathered}
$$

Las anteriores son cargas de diferente magnitud, por lo que se puede diseñar indistintamente un solo tipo de rodamiento para los dos apoyos. Para esto se calcula la capacidad de carga dinámica mediante la relación:

$$
\left.L_{10}=\frac{1000000}{60 n} \varepsilon_{P_{r}^{p}}^{p}\right)
$$

Entonces el valor de seguridad de carga está dado por:

$$
\frac{C}{P_{r}}=4
$$

Puesto que el rodamiento está sometido a carga radial pura $P_{r}=R a=2437 \mathrm{~N}$

$\mathrm{C}>8 \mathrm{P}=9748 \mathrm{~N}$

Para un diámetro de $50 \mathrm{~mm}$ se elige el rodamiento con soporte, con designación F210. 
Proyecto de diseño y construcción de una cámara de transporte, tipo tornillo para biomasa forestal

Se presenta la construcción de la cámara de transportación a partir de los parámetros establecidos en la etapa de diseño, tomando en cuenta el cumplimiento de la normativa ASME vigente, de manera que, los procesos estén dentro de los parámetros técnicos, garantizando eficiencia y resultados óptimos: (TABLA 2 Y 3)

\section{Construcción Tornillo}

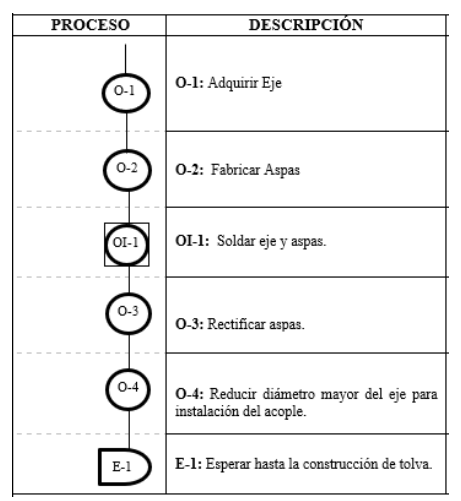

Tabla 2 Proceso de Construcción Tornillo

\section{Construcción cámara:}

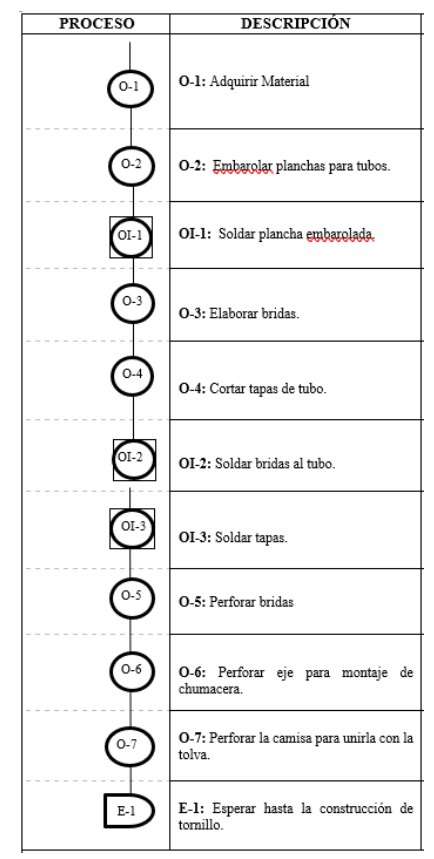

Tabla 3 Proceso de Construcción Cámara 


\section{Desarrollo}

Se construye una cámara de transportación con una capacidad de $25.7 \mathrm{~kg}$ (figuras 9), que traslada biomasa de manera continua y eficiente.

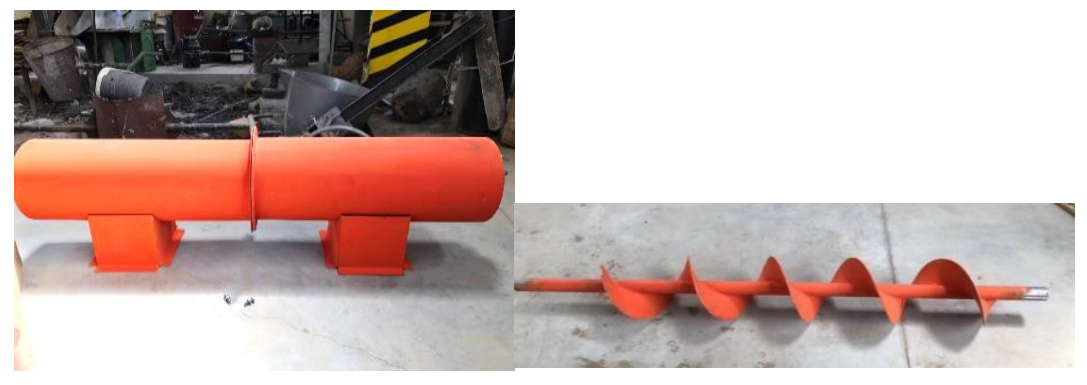

Figura 9. Camisa y Tornillo

Se reduce el tiempo de trabajo de operarios en el proceso de alimentación de biomasa, al realizarse directamente con la cámara construida.

\section{Conclusiones}

En el estudio de transporte de desechos sólidos se determinó que los transportadores de sólidos volumétricos son utilizados principalmente en la agricultura, siendo los transportadores de tornillo los equipos más sencillos y económicos que existen para mover sólidos de un lugar a otro de cualquier instalación. Con la aplicación de este tipo de transportadores existe basta evidencia de que se obtiene varias ventajas como: eficiencia del proceso, disminución de daños en los equipos, disminución en las horas de trabajo de los operarios, entre otras.

Al considerar que la principal actividad es transportar biomasa forestal a una velocidad de $25,71 \mathrm{~kg} / \mathrm{h}$ se determina la utilización de un transportador basado en un tornillo sin fin, puesto que permite desplazar partículas sólidas y medir la dosificación mediante la velocidad del motor que locontrola.

Se construye un transportador de biomasa forestal que alimente de forma continua, automática y rápida al gasificador tipo Downdraft, cumpliendo con las necesidades descritas por el usuario las cuales fueron identificadas a través de la metodología de diseño conocida como la "Casa de la Calidad". 
Proyecto de diseño y construcción de una cámara de transporte, tipo tornillo para biomasa forestal

Tras la ejecución de pruebas de funcionamiento se determinó que con el uso del transportador se disminuye el tiempo de interacción humana en la alimentación de materia prima al realizar una sola recarga para todo el proceso.

\section{Referencias Bibliográficas}

Carrillo, Angélica. 2015. Diseño y simulación de una planta dosificadora automática de suplemento nutricional para ganado lechero. Quito: ESPN, 2015.

Franco, Luis, Galicia, Jesús y Ostria, Diana. 2010. Desarrollo de un Sistema de Dosificación Autómático de Alimento para Equinos. México DF: s.n., 2010.

Morales, Emilio. 2010. DIiseño y construcción de un dosificador y empacador de producto alimenticio molido y seco de 50 gr. Latacunga: ESPE, 2010.

Orozco, Edisson y Guerrero, Víctor. 2016. Diseño y montaje de un sistema dosificador para galletas tipo Cracker fermentadas. Pereira: Universidad Tecnológica de Pereira, 2016.

Osipna, John y Taborda, Diego. 2013. Diseño y Construcción de un prototipo de un Dosificador de Alimento concentrado para Cerdos. Pereira: s.n., 2013.

Rivera, César. 2002. Diseño y construcción de un dosificador de tres productos en forma secuencial. Quito: Escuela Politécnica Nacional, 2002.

Sanz, Rodrigo, Oliveros, Carlos y Peñuela, Aida. 2014. Avances Técnicos Cenicafé. Bogotá: Federación Nacional de Cafeteros de Colombia, 2014. Instalación de Separador Hidráulico.

TECNIDOS: Sistemas y Procesos. 2014. Transportadores de Sólidos. Transportadores de Sólidos. [En línea] TECNIDOS, 8 de 10 de 2014. [Citado el: 7 de junio de 2017.] http:/tecnidos.com/index.php/productos/dosificacion-de-solidos/transportadores-de-solidos-2/. 